\title{
A multi-centre cohort study evaluating the role of inflammatory markers in patient's presenting with acute ureteric colic (MIMIC)
}

\author{
T.T. Shah ${ }^{\mathrm{a}, *}$, A.G. O’Keeffe ${ }^{\mathrm{b}}$, C. Gao ${ }^{\mathrm{a}}$, T. Manning ${ }^{\mathrm{c}}$, A. Peacock ${ }^{\mathrm{d}}$, S. Cashman ${ }^{\mathrm{a}}$, A. Nambiar ${ }^{\mathrm{a}}$, B. Lamb $^{\mathrm{a}}$, \\ M. Cumberbatch ${ }^{a}$, R. Pickard ${ }^{\text {e }}$, P. Erotocritou ${ }^{f}$, D. Smith ${ }^{\mathrm{g}}$, V. Kasivisvanathan ${ }^{\mathrm{a}}$, BURST Collaborative \\ MIMIC Study Group
}

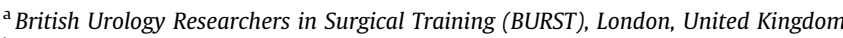

${ }^{\mathrm{b}}$ University College London, Dept. of Statistical Science, London, United Kingdom

${ }^{\mathrm{c}}$ Australian Young Urology Researchers Organisation (YURO), Australia

${ }^{\mathrm{d}}$ Information Services Division, University College London (UCL), United Kingdom

e Newcastle University, Dept. of Urology, London, United Kingdom

${ }^{\mathrm{f}}$ Whittington Hospital, Dept. of Urology, London, United Kingdom

${ }^{\mathrm{g}}$ University College London Hospital, Dept. of Urology, London, United Kingdom
}

\section{A R T I C L E I N F O}

\section{Article history:}

Received 19 August 2017

Received in revised form 26 September

2017

Accepted 26 September 2017

Available online 28 September 2017

\section{Keywords:}

Urolithiasis

Inflammation

Spontaneous stone passage

Predictive model

White cell count

\begin{abstract}
A B S T R A C T
Background: Spontaneous Stone Passage (SSP) rates in acute ureteric colic range from 47 to $75 \%$. There is conflicting evidence on the role of raised inflammatory markers in acute ureteric colic. The use of an easily applicable biomarker that could predict SSP or need for intervention would improve the management of obstructing ureteric stones. Thus, there is a need to determine in an appropriately powered study, in patients who are initially managed conservatively, which factors at the time of acute admission can predict subsequent patient outcome such as SSP and the need for intervention. Particularly, establishing whether levels of white cell count (WBC) at presentation are associated with likelihood of SSP or intervention may guide clinicians on the management of these patients' stones.

Design: Multi-center cohort study disseminated via the UK British Urology Researchers in Surgical Training (BURST) and Australian Young Urology Researchers Organisation (YURO).

Primary research question: What is the association between WBC and SSP in patients discharged from emergency department after initial conservative management?

Patient population: Patients who have presented with acute renal colic with CT KUB evidence of a solitary ureteric stone. A minimum sample size of 720 patients across 15 centres will be needed. Hypothesis: A raised WBC is associated with decreased odds of spontaneous stone passage.

Primary outcome: The occurrence of SSP within six months of presentation with acute ureteric colic (YES/ NO). SSP was defined as absence of need for intervention to assist stone passage.

Statistical analysis plan: A multivariable logistic regression model will be constructed, where the outcome of interest is SSP using data from patients who do not undergo intervention at presentation. A random effect will be used to account for clustering of patients within hospitals/institutions. The model will include adjustments for gender, age as control variables.

(C) 2017 The Authors. Published by Elsevier Ltd on behalf of Surgical Associates Ltd. This is an open access article under the CC BY-NC-ND license (http://creativecommons.org/licenses/by-nc-nd/4.0/).
\end{abstract}

\section{Introduction/background}

Urolithiasis is a very common problem with a lifetime incidence of up to $19 \%$ in men and $8 \%$ in women. In a high-income European Country, studies report over 750,000 cases a year with $25 \%$ experiencing recurrence [1-3]. The American Urology Association and European Association of Urology guidelines report a $68 \%$

\footnotetext{
* Corresponding author.

E-mail address: taimur.shah@ucl.ac.uk (T.T. Shah).
}

spontaneous ureteral stone passage rate for stones $<5 \mathrm{~mm}$ and 47\% for stones between 5 and $10 \mathrm{~mm}$ [4]. Median time to stone passage was 39-40 days for stones less than $10 \mathrm{~mm}$. In patients who do not have immediate intervention on presentation, there is uncertainty as to when intervention is necessary, if at all. The use of an easily applicable biomarker that could predict spontaneous stone passage or need for intervention would improve the management of obstructing ureteric stones.

There is conflicting evidence on the role of raised inflammatory markers in acute ureteric colic with some studies showing raised 
inflammatory markers are associated with an improved stone passage rate whilst others claiming a lower rate with an increased need for intervention. After a multivariable analysis, Sfoungaristos found a statistically significant increased chance of stone passage in patients with a raised WBC or neutrophil count [5]. They reasoned that as both WBC and neutrophils are general markers of inflammation they could represent the inflammation caused to the ureter by the stone passage and suggested that stones that do pass along the length of the ureter may have greater potential to cause inflammation.

However, Park et al., showed directly conflicting results, where the stone passage rate was $11.4 \%$ higher in those with a normal neutrophil count when compared to those with a raised count, defined as $>74 \%$ [6]. The authors also found a decreased spontaneous stone passage rate with increasing CRP levels (high group defined as $>10 \mathrm{mg} / \mathrm{dl}$ ). They reasoned that the inflammatory response leads to oedema and ureteric convulsion which prevent spontaneous stone passage. Others have also found a negative correlation with raised CRP and spontaneous stone passage and Angulo et al. found an increased need for intervention in those with a raised WBC and/or CRP [7,8].

Given the conflicting evidence, there is a need to determine in an appropriately powered study, in patients who are initially managed conservatively, which factors at the time of acute admission can predict subsequent patient outcome such as spontaneous stone passage and the need for intervention. Particularly, establishing whether levels of white cell count at presentation are associated with likelihood of spontaneous passage or intervention may guide clinicians on the management of these patients' stones. This study will also allow us to determine regional variations in stone management, highlighting areas for guideline development.

The study was designed to allow reporting according to the STROBE guidelines (The Strengthening the Reporting of Observational Studies in Epidemiology (STROBE) statement: guidelines for reporting observational studies) [9].

\section{Methods and analysis}

\subsection{Design}

Multi-center cohort study disseminated via the UK British Urology Researchers in Surgical Training (BURST) and Australian Young Urology Researchers Organisation (YURO).

Emergency department records, follow up records and surgical entry notes will be assessed retrospectively for all consecutive patients at the site who met the eligibility criteria (See Section 2.4). Patients are followed up for 6 months or until they experience spontaneous stone passage or intervention. Follow-up data is defined as verifiable information on patient attendance to either an outpatient clinic, imaging test or admission for intervention.

Phase 1: A pilot study in 120 patients at 6 sites (20 per site). This phase has been completed.

Pilot outcome measures included 1. Ease of subject selection, 2. Availability of data, 3. Ease of use of data collection tool, 4. Suitability of variables selected and 5 . Time taken per patient.

The pilot study revealed adequate data on selected variables was routinely recorded retrospectively. The data collection tool was refined for use in the main phase of the study.

Phase 2: Multi-centre study in at least 720 patients in at least 15 sites.

\subsection{Primary research question}

What is the association between White Cell Count (WBC) and spontaneous stone passage (SSP) in patients discharged from emergency department after initial conservative management?
SSP was defined as absence of need for intervention to assist stone passage, in line with landmark studies in this field [10].

\subsection{Secondary research questions}

I. What is the association between CRP and SSP in patients presenting with acute ureteric colic?

II. What is the WCC and CRP threshold associated with the lowest SSP rate?

III. What is association between medically expulsive use (MET) and SSP?

IV. What are the strongest predictors of spontaneous stone passage in patients with acute ureteric colic when adjusting for key confounding variables?

$\mathrm{V}$. Is there regional variation in the management of patients presenting with acute ureteric colic?

\subsection{Patient population}

Patients who have presented with acute renal colic who have CT KUB evidence of a solitary ureteric stone.

Exclusion criteria include: patients with non-obstructing renal stones, patients with multiple ureteric stones and patients who have second or subsequent presentation of a previous stone.

\subsection{Hypothesis}

A raised WCC is associated with decreased odds of spontaneous stone passage.

\subsection{Follow up}

Patients will be followed up for at least 6 months or until they experience spontaneous stone passage or intervention.

\subsection{Power calculation}

Outcome variable defined as:

$1=$ Stone-free

$0=$ Not stone free

As per the accepted criteria of 10 events per variable (EPV) set up by Peduzzi et al. for logistic regression analyses and making that assumption that 0.25 is the rate of the "rarer event" (25\% not stone free - amongst those who were discharge with conservative management) our sample size was deemed as being:

- $\mathrm{m}=$ number of model parameters for explanatory variable effects.

- $r=$ estimated overall rate of intervention/non-passage of stone. Then, approximately, the total number of patients required is given by:

$\mathrm{N}=(10 \times \mathrm{m}) / \mathrm{r}=(10 \times 18) / 0.25=720$ patients

Thus, a sample size of 720 patients will be sufficient.

\section{Statistical analysis plan}

Analysis will follow a pre-specified statistical analysis plan, with no planned interim analyses.

\subsection{Organisation of data}

Release of collected data shall occur after the final participant has completed follow-up, once all data have been entered onto the database and checked and the SAP has been finalised and approved 
Prior to performing analyses, data validation shall be performed on the collected data on a site-by-site basis, by a member of the MIMIC Study Group independent to that site. To ensure high quality data, each primary and secondary outcome variable will be checked for:

- missing values;

- values beyond an acceptable range;

- consistency between different variables;

- date consistencies

- other inconsistencies.

In addition, $10 \%$ of all patient data for each site will be checked at random for sense and consistency. The findings of data quality assessment will be documented with full audit record.

If missing values or other inconsistencies are present, then queries will be sent to the site to address.

\subsection{Summary of outcome measures}

Variables of interest will be summarised at presentation. The term 'presentation' refers to a patient's entry to the cohort, in other words, the visit at which he/she first presents with acute ureteric colic and is eligible to enter the cohort.

We shall provide statistical summaries for the following variables at presentation:

a) Hospital/Institution site

b) Year of presentation

c) Age (in years)

d) Gender (male/female)

e) Previous stone history (YES/NO)

f) Stone size (in $\mathrm{mm})^{*}$

g) Stone side (right or left)*

h) Stone location (proximal ureter, mid ureter, distal ureter)*

i) White blood cell count (WBC) (in $\left.\times 10^{9} / \mathrm{L}\right)$

j) Neutrophil count (in $\left.\times 10^{9} / \mathrm{L}\right)$

k) C-Reactive protein (CRP) level (in $\mathrm{mg} / \mathrm{L}$ )

1) Creatinine (umol/L)

m) Hydronephrosis (YES/NO*

n) Hydroureter (YES/NO)*

o) Peri-nephric stranding (YES/NO)*

p) Administration of NSAID (YES/NO)

q) Administration of Medically Expulsive Therapy (MET) (YES/NO)

r) Administration of antibiotics (YES/NO)

*As reported by the radiologist

\subsection{Primary outcome}

The occurrence of 'spontaneous stone passage' within six months of presentation with acute ureteric colic (YES/NO). Spontaneous stone passage (SSP) was defined as absence of need for intervention to assist stone passage.

\subsection{Secondary outcomes}

- Mean white cell count (in $\left.\times 10^{9} / \mathrm{L}\right)$

- Mean C-reactive protein $(\mathrm{mg} / \mathrm{L})$

- Proportion of patients who have immediate intervention

- Proportion of patients who have subsequent intervention during follow up

- Proportion of patients who have ureteroscopy/cystoscopy and JJ stenting/nephrostomy
- Proportion of patients who are lost to follow up after initial presentation

- Mean stone size (mm)

- Proportion of patients with upper/mid/distal ureteric stones

- Proportion of patients having medical expulsive therapy

- Proportion of patents having antibiotics

- Proportion of patients having NSAIDs

- Proportion of patients with renal impairment

\subsection{Statistical analyses}

\subsubsection{Description of demographic variables at presentation}

Continuous variables will be summarised by reporting the mean, standard deviation and range. Categorical variables will be summarised using frequency tables.

Summaries will be shown for all patients and stratified by whether or not a patient underwent an intervention at presentation (i.e. before discharge).

\subsubsection{Loss to follow-up}

A descriptive summary of patients lost to follow-up shall be made.

For patients lost to follow-up we all participating centers will be advised to make adequate arrangements to contact patients. From the pilot, it was found that this was dependent on the local infrastructure but often involved a telephone calls and/or letters to patients/GP.

\subsubsection{Primary outcome analyses}

Immediately after presentation, patients shall be classified as either having (1) undergone intervention at presentation (i.e. before discharge) or (2) discharged shortly after presentation with no further intervention at that point. The number of patients who undergo intervention at presentation shall be reported.

The group of patients for whom the primary outcome is of most interest is those who are discharged after presentation without, at that time, any intervention. For this group:

A multivariable logistic regression model will be constructed, where the outcome of interest is 'spontaneous stone passage within six months' using data from patients who do not undergo intervention at presentation. A random effect will be used to account for clustering of patients within hospitals/institutions. The multivariable model will include adjustments for gender, age as control variables.

Univariable logistic regression models will be used to determine the level of association between other variables of interest (reported in Section 3.2) and spontaneous stone passage within six months. Variables for which there is a significant association with spontaneous stone passage in the corresponding fitted univariable model will be included in final multivariable model for spontaneous stone passage. A significant association is determined by a P-value $<0.05$ in a hypothesis test pertaining to the corresponding model coefficient, after accounting for multiple hypothesis testing using a Bonferroni correction).

Through fitting this model, the main aim is to a make inference on the variables that influence the probability of 'spontaneous stone passage' within six months of presentation. Odds ratios, related to the probability of spontaneous stone passage, shall be reported for each explanatory variable. The predictive ability of the final model shall be assessed using ten-fold cross-validation.

\subsubsection{Secondary outcome analyses}

Continuous secondary outcome variables will be modelled using mixed effects liner models (with transformations of variables performed, where appropriate). Binary secondary outcome 
variables will be modelled using mixed effects logistic regression models.

\subsubsection{Other exploratory analyses}

- Consideration will be given to a time to event analysis investigating the association of white cell count and spontaneous stone passage from initial presentation with acute renal colic to spontaneous stone passage.

- Further Cox and logistic regression models that include other variables measured at presentation (e.g. stone size, neutrophil count) shall be considered, as both univariable models and for inclusion in multivariable models.

- A range of investigatory inflammatory markers will be recorded at baseline and their role in acute renal colic and spontaneous stone passage may be explored in subsequent studies.

\subsubsection{Missing data}

An adjustment for missing explanatory variables will be considered in the analysis of the primary outcome only. Baseline characteristics of patients with and without missing values will be compared. Depending on the extent of missingness, the predictors of missing values will be identified the primary outcome analysis will be adjusted for those predictors of missing values which are related to missingness. A sensitivity analysis of the primary outcome that accounts for missing data shall be considered using multiple imputation (11).

\section{Ethics}

All local institutional governance processes will be followed prior to carrying out the project at that site. Where ethical approval is required this shall be obtained. Where research and development departments grant ethical exemption for this service evaluation project, registration numbers, when granted, will be kept centrally.

\section{Discussion}

Urolithiasis is a common problem that is currently on the rise in the some developed countries [11]. During the acute admission there is some consensus as to which patients warrant urgent immediate intervention (e.g. those with sepsis). However it is less clear how to manage the large cohort of patients who are initially managed conservatively and do not require immediate intervention. Specifically, quantifying the probability of spontaneous stone passage and thus determining the need for intervention is challenging. A biomarker that could be measured on first presentation that could predict spontaneous stone passage would help guide management of these patients. Inflammatory markers, such as white cell count, would be attractive biomarkers, though there is conflicting evidence on the role of raised inflammatory markers in acute ureteric colic and spontaneous stone passage, with previous studies also either lacking power and/or not adjusting for important confounding factors.

MIMIC aims to show whether there is any association between WBC on admission and spontaneous stone passage in patients followed up for 6 months. Other potential confounders of SSP will be adjusted for. These data will be used by to develop a risk calculator/model predicting spontaneous stone passage in patients presenting with acute ureteric colic. Such a calculator would allow a more informed decision making process with patients regarding their management plans.

In addition, regional variation in the follow-up and management of patients admitted with acute ureteric colic will be determined. There are also a group of patients who are deemed lost to follow-up and they warrant further investigation as they are at risk of potential chronic renal injury from a prolonged silently obstructing stone. It will be assessed whether institutional protocols are robust enough to ensure good follow up of these patients.

MIMIC aims to provide an evidence base that can guide clinicians in the management of patients with acute renal colic. MIMIC data may lead to the design of interventional studies, which can be used to improve the management of patients with acute renal colic.

\section{Authors' contributions}

Study conception and design: TTS, VK, AOK.

Study coordination and delivery: TTS, CG, AN, SC, TM, AP.

Acquisition of data: All members of the BURST Collaborative MIMIC Study Group.

Statistical analysis: TTS, VK, AOK.

Analysis and interpretation of data: TTS, VK, AOK.

Drafting of manuscript: TTS, VK, AOK.

Critical revision: DS, PE, BL.

\section{Source of funding}

The BURST Research Collaborative has previously received funding from the British Association of Urological Surgeons, British Journal of Urology International and The Urology Foundation.

\section{Conflicts of interest statement}

TTS would like to acknowledge funding from the St Peters Trust for clinical research and has received funding for conference attendance from Astellis, Ferring and Galil Medical.

VK is funded by a Doctoral Research Fellowship from the National Institute for Health Research. The views expressed in this publication are those of the authors and not necessarily those of the NHS, the National Institute for Health Research or the Department of Health. VK has previously received research funding from the European Association of Urology and the American Urology Association.

All others authors declare no conflicts of interest.

\section{References}

[1] W.L. Strohmaier, Socioeconomic aspects of urinary calculi and metaphylaxis of urinary calculi, Urologe A 39 (2) (2000) 166-170.

[2] A. Hesse et al., Study on the prevalence and incidence of urolithiasis in Germany comparing the years 1979 vs. 2000, Eur. Urol. 44 (6) (2003) 709-713.

[3] A. Trinchieri, Epidemiology of urolithiasis: an update, Clin. Cases Miner. Bone Metab. 5 (2) (2008) 101-106.

[4] A. Skolarikos et al., The role for active monitoring in urinary stones: a systematic review, J. Endourol. 24 (6) (2010) 923-930.

[5] S. Sfoungaristos, Role of white blood cell and neutrophil counts in predicting spontaneous stone passage in patients with renal colic, BJU Int. 110 (8 Pt B) (2012), p. E339-45.

[6] C.H. Park et al., Relationship between spontaneous passage rates of ureteral stones less than $8 \mathrm{~mm}$ and serum C-reactive protein levels and neutrophil percentages, Korean J. Urol. 54 (9) (2013) 615-618.

[7] H.A. Aldaqadossi, Stone expulsion rate of small distal ureteric calculi could be predicted with plasma C-reactive protein, Urolithiasis 41 (3) (2013) 235-239.

[8] J.C. Angulo et al., The value of C-reactive protein determination in patients with renal colic to decide urgent urinary diversion, Urology 76 (2) (2010) 301 306.

[9] E. von Elm et al., Strengthening the reporting of observational studies in epidemiology (STROBE) statement: guidelines for reporting observational studies, BMJ 335 (7624) (2007) 806-808.

[10] R. Pickard et al., Medical expulsive therapy in adults with ureteric colic: a multicentre, randomised, placebo-controlled trial, Lancet 386 (9991) (2015) 341-349.

[11] B.W. Turney et al., Trends in urological stone disease, BJU Int. 109 (7) (2012) 1082-1087. 